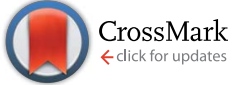

Cite this: J. Mater. Chem. C, 2014, 2, 10468

\title{
Highly luminescent dual mode rare-earth nanorod assisted multi-stage excitable security ink for anti- counterfeiting applications $\uparrow$
}

\begin{abstract}
Pawan Kumar, Jaya Dwivedi and Bipin Kumar Gupta*
In this paper, the synthesis of multi-stage excitable $(379 \mathrm{~nm}, 980 \mathrm{~nm}$ and $1550 \mathrm{~nm})$, highly luminescent $\mathrm{Y}_{2} \mathrm{O}_{3}: \mathrm{Yb}^{3+} / \mathrm{Er}^{3+}$ nanorods by a hydrothermal method which can emit both hypersensitive green (562 $\mathrm{nm}$ ) and strong red $(660 \mathrm{~nm})$ in a single host lattice and which can be used for a transparent security ink application is reported. Furthermore, these luminescent nanorods also exhibit both down-shift (1127 nm) as well as upconversion ( $562 \mathrm{~nm}$ ) features at a single excitation near infrared wavelength of $980 \mathrm{~nm}$. The characterization of these luminescent nanorods was explored by studying their structure/microstructure, using photoluminescence and time-resolved spectroscopic techniques. Furthermore, these luminescent nanorods exhibited the tuning of emission colours from red to green by controlling the sintering temperature. It was demonstrated that these novel luminescent nanorods offer new opportunities for making high-end, multi-stage excitable transparent security ink. The state-of-art production process of ink is easy and it is most suitable for bulk production at an economical cost. Thus, the security inks could be used globally in applications for protection against counterfeiting.
\end{abstract}

Received 14th September 2014
Accepted 20th October 2014

DOI: $10.1039 / \mathrm{c} 4 \mathrm{tc0} 2065 \mathrm{k}$

www.rsc.org/MaterialsC country has opted for protection against fake currencies by either use of holographic techniques or by use of visible luminescent ink or bands and so on (which glow under ultraviolet (UV) light). ${ }^{6-10}$ Furthermore, commercial and industrial sectors, particularly those producing branded goods and pharmaceuticals, have also adopted these techniques and have benefitted tremendously from these safety measures against counterfeiting. ${ }^{11,12}$ Besides this, another area of security where a more overt colorimetric indicator can be used is food security, where sensing the presence of oxygen is a principal goal - the area of intelligent packaging. ${ }^{13}$

During the past decades, a number of anti-counterfeiting technologies such as simple markers, plasmonic security labels, holograms, security inks and so on, were developed as a shield against counterfeiting. ${ }^{10,14}$ Today, the development of new materials for anti-counterfeiting is of great importance for their use in protection and detection of counterfeiting. However, from the security point of view, the luminescent materials provide improved security because of their unique physical, chemical and optical properties. Although there are many semiconductor nanoparticles such as cadmium sulfide, cadmium selenide, cadmium telluride and so on, which have been reported to be used as luminescent security ink, they have their own disadvantages such as toxicity, broad emission bands and solubility in harmful solvents. The formation of a stable and transparent solution of these nanoparticles is also difficult. Furthermore, the lanthanide doped phosphors have many advantages over these materials such as low toxicity, sharp emission bands and solubility. Therefore, the lanthanide-based 
transparent security ink has become a successful tool for security labels, identification markers and barcode systems.

Our earlier efforts were to develop red emitting down-shift invisible security ink based on polyvinyl alcohol (PVA) as well as sodium hexametaphosphate (SHMP) medium and these were used successfully to demonstrate their use in terms of security coding. ${ }^{2}$ Efforts are being made by several groups to develop either upconversion or downconversion security ink based on lanthanide materials., ${ }^{\mathbf{4} 5}$ Only a single stage coding capability has been achieved so far by using these luminescent materials. This implies that the luminescent fluorophores developed can be excited by a single wavelength, which subsequently emits only at a single wavelength. This coding technique appears to be comparatively less effective at protecting documents. Because of this reason, a high performance, multi-stage coding with many excitations and at least two colour emissions has been awaited for a long time. To develop a phosphor with all the desirable properties at an economical cost is a great challenge in this area which is being addressed in the present investigations. Now in the present research, our purpose was to develop a transparent security ink with multi-stage coding in a single host based on a highly luminescent nanostructure which is not only economic but also beneficial for high end protection. Earlier, several attempts had been made but their protection techniques were common and utilized single stage protection. Even nowadays, many groups are trying to use the dual mode emission by incorporating two or more kinds of activator in a single host, although their intention was entirely different, because incorporating many activators is an expensive technique and dispersion is also an important issue. ${ }^{15}$ A single host lattice based luminescent material with single activator which can emit both upconversion as well as down-shift simultaneously has been developed in the current research. As a result, it provides multi-stage coding in a host. Furthermore, the choice of the material for security ink applications depends upon the physical and chemical properties as well as the morphology of nanomaterials. The yttrium oxide $\left(\mathrm{Y}_{2} \mathrm{O}_{3}\right)$ is an attractive host for security ink applications because of its physical, chemical and optical properties. An additional advantage of using nanorods is that it also helps to bring down the amount of material required for their use in security ink applications, thus reducing the production cost and as well the rod shape is good in fillers in a cellulose ink matrix. ${ }^{16}$ On the basis of this concept, highly luminescent nanorod-based, multi-stage excitable and transparent inks for security applications were developed.

In this study, the development of multi-stage excitable (379 $\mathrm{nm}, 980 \mathrm{~nm}$ and $1550 \mathrm{~nm}$ ), highly luminescent $\mathrm{Y}_{2} \mathrm{O}_{3}: \mathrm{Yb}^{3+} / \mathrm{Er}^{3+}$ nanorods was demonstrated by using a hydrothermal technique for security inks, which can intensely emit hypersensitive green and strong red colours. Furthermore, these nanorods have also shown both down-shift as well as having an upconversion nature for a single excitation wavelength at $980 \mathrm{~nm}$ which has been reported for such applications. The gross structural, morphological, and microstructural investigations of these luminescent nanorods have been carried out through X-ray diffraction, scanning electron microscopy and high-resolution transmission electron microscopy/transmission electron microscopy techniques. The spectroscopic characterizations of these luminescent nanorods have been done through photoluminescence and time-resolved spectroscopic techniques. The promising use of this luminescent nanorod-based transparent security ink for security codes on black paper using a standard screen printing technique as well as security spot marking on currency for a high end protection application which exhibits a new paradigm shift in luminescent security ink applications was also demonstrated.

\section{Experimental section}

The precursors: $\mathrm{Y}_{2} \mathrm{O}_{3}$ (99.99\%), erbium nitrate pentahydrate $\left(\mathrm{Er}\left(\mathrm{NO}_{3}\right)_{3} \cdot 5 \mathrm{H}_{2} \mathrm{O}, 99.99 \%\right)$, ytterbium(III) chloride hexahydrate $\left(\mathrm{YbCl}_{3} \cdot 6 \mathrm{H}_{2} \mathrm{O}, 99.99 \%\right)$ and $N$-cetyl- $N, N, N$ trimethylammonium bromide $\left(\mathrm{C}_{19} \mathrm{H}_{42} \mathrm{BrN}\right.$, CTAB $)$ were purchased from SigmaAldrich. All reagents were of analytical (AR) grade and used as received without further purification. Double distilled water was used throughout the experiments.

The $\mathrm{Y}_{2} \mathrm{O}_{3}: \mathrm{Yb}^{3+} / \mathrm{Er}^{3+}$ nanorods were synthesized using a hydrothermal method. The precursors used were: $\mathrm{Y}_{2} \mathrm{O}_{3}$, $\mathrm{Er}\left(\mathrm{NO}_{3}\right)_{3} \cdot 5 \mathrm{H}_{2} \mathrm{O}, \mathrm{YbCl}_{3} \cdot 6 \mathrm{H}_{2} \mathrm{O}$ and CTAB. The nanorods were doped with an optimized concentration of rare earth ions (1 mol\% of Er and $2 \mathrm{~mol} \%$ of $\mathrm{Yb}$ ). The stoichiometric amount of $\mathrm{Y}_{2} \mathrm{O}_{3}$ was dissolved in nitric acid followed by stirring at $80{ }^{\circ} \mathrm{C}$ until the solution turned into a transparent nitrate solution. The precursors $\operatorname{Er}\left(\mathrm{NO}_{3}\right)_{3} \cdot 5 \mathrm{H}_{2} \mathrm{O}, \mathrm{YbCl}_{3} \cdot 6 \mathrm{H}_{2} \mathrm{O}$ and CTAB were dissolved in deionized water. The prepared solutions were mixed in ethanol solution (1: 5 ethanol : water) under vigorous stirring at room temperature. The sodium hydroxide solution was added to the above mixture and then stirred magnetically until the $\mathrm{pH}$ of the final solution became $\sim 12$. Several runs were made using different $\mathrm{pH}$ values and ultimately, it was found that a $\mathrm{pH}$ of $\sim 12$ favoured the reaction which produced long uniform nanorods. Then this solution was transferred into a sealed hydrothermal bomb which was kept at $185{ }^{\circ} \mathrm{C}$ for 10 hours in a box furnace. The precipitate obtained was centrifuged several times with de-ionized water at $5000 \mathrm{rpm}$ and then dried at $100{ }^{\circ} \mathrm{C}$. The final product was heated at $1000^{\circ} \mathrm{C}$ in a box furnace for 6 hours. In this method, the yield of material was more than $90 \%$ with a high degree of homogeneity throughout the sample.

A standard polyvinyl chloride (PVC) gold medium (locally fabricated, printing ink manufactured by Commercial Techno Colours, Ram Nagar, Varanasi 221005, India) was used to disperse the as-synthesized dual mode $\mathrm{Y}_{2} \mathrm{O}_{3}: \mathrm{Yb}^{3+} / \mathrm{Er}^{3+}$ nanorods before use as security printing application. The commercial PVC gold medium used in the present investigation is shown in Fig. S1 (see $\mathrm{ESI}_{\dagger}^{\dagger}$ ). The choice of dispersive medium is important for two purposes, firstly to disperse rare earth metals into the medium without formation of clusters or agglomerates of nanorods and secondly, for obtaining better printing, the viscosity of the medium $(3000 \mu \mathrm{P})$ is very important as it provides the sticky nature of the printing paper. Our earlier efforts using PVA as medium failed to avoid the formation of clusters. ${ }^{2}$ In a typical experiment, $50 \mathrm{~mL}$ of PVC gold medium solution was placed in a beaker and $200 \mathrm{mg}$ of $\mathrm{Y}_{2} \mathrm{O}_{3}: \mathrm{Yb}^{3+} / \mathrm{Er}^{3+}$ 
nanorods were mixed with the solution. The resultant mixture was mixed ultrasonically at $45 \mathrm{kHz}$ for $30 \mathrm{~min}$ to obtain a good dispersion. The highly transparent $(\sim 81 \%)$ colloidal solution was obtained which is shown in Fig. S2 (see ESI†). To make a pattern onto black paper, a standard screen printing technique for object printing as well as for alphabets was used.

The gross crystal structure analysis of the samples was carried by using X-ray powder diffraction (XRD) with a Bruker AXS D8 Advance X-ray diffractometer, using $\mathrm{Cu} \mathrm{K}_{1}$ radiation $(\lambda=1.5406 \AA)$. The UV absorption spectra were recorded using a Shimadzu UV 160 UV-visible spectrometer. Thermogravimetric analysis was done using a thermal analysis instrument with a heating rate of $10{ }^{\circ} \mathrm{C} \mathrm{min}{ }^{-1}$ in an air flow of $100 \mathrm{~mL} \mathrm{~min}{ }^{-1}$. Raman studies were carried out using a Renishaw inVia Raman spectrometer, with an excitation source of $785 \mathrm{~nm}$. The surface morphology and energy dispersive X-ray (EDAX) was examined using a Carl ZEISS EVO 18 scanning electron microscope with $10 \mathrm{kV}$ operating voltage. Transmission electron microscopy (TEM) and high-resolution transmission microscopy (HRTEM) micrographs were recorded using a Tecnai G2 S-Twin scanning transmission electron microscope with a field emission gun operating at $300 \mathrm{kV}$. The photoluminescence (PL) and timeresolved spectroscopy were carried out using an Edinburgh Instruments spectrometer, where a xenon lamp acts as the source of excitation. The PL mapping of luminescent nanorods was performed using a WITech alpha $300 \mathrm{R}+$ confocal PL microscope system, where 375, 980 and $1550 \mathrm{~nm}$ diode lasers act as a source of excitation. The near infrared (NIR) emissions above $1100 \mathrm{~nm}$ were recorded using a NIR detector. The fluorescent images were recorded using a Nikon ECLIPSE 80i microscope.

\section{Results and discussion}

A novel strategy was adopted to synthesize highly luminescent $\mathrm{Y}_{2} \mathrm{O}_{3}: \mathrm{Yb}^{3+} / \mathrm{Er}^{3+}$ nanorods using a customized hydrothermal method. The proper interaction between the dopant $\left(\mathrm{Yb}^{3+}\right)$ and co-dopant $\left(\mathrm{Er}^{3+}\right)$ is very important to obtain highly efficient luminescent materials. Here, the optimization of the concentration played a key role in getting bright PL material. The addition of the $\mathrm{Yb}^{3+}$ ion increases the absorption because the $\mathrm{Yb}^{3+}$ ion efficiently absorbs the energy and becomes excited. The excited $\mathrm{Yb}^{3+}$ ion transfers this energy to the $\mathrm{Er}^{3+}$ ion which promotes its own electrons in a higher energy level as shown in Fig. S3 (see ESI $\dagger$ ). Subsequently, the population in the excited states of the $\mathrm{Er}^{3+}$ increases significantly. The energy transfer depends upon the doping concentration of both dopant as well as co-dopant. The main focus of the present investigation is to optimize the green PL emission intensity of $\mathrm{Y}_{2} \mathrm{O}_{3}: \mathrm{Yb}^{3+} / \mathrm{Er}^{3+}$ nanorods while keeping the concentration of $\mathrm{Yb}^{3+}$ fixed. The $\mathrm{PL}$ intensity of the green emission was varied with respect to the change in concentration of $\mathrm{Er}^{3+}$ from 0.5 to $2.5 \mathrm{~mol} \%$ at a fixed concentration of $\mathrm{Yb}^{3+}(2 \mathrm{~mol} \%)$ (see Fig. S4 in the ESI†). The optimum concentration of $\mathrm{Er}^{3+}$ was shown to be $\sim 1 \mathrm{~mol} \%$. Furthermore, the optimum concentration of $\mathrm{Yb}^{3+}$ was also investigated from $1-3 \mathrm{~mol} \%$ at a fixed optimum concentration of $\mathrm{Er}^{3+}$ of $\sim 1 \mathrm{~mol} \%$. It was found that the efficient energy transfer with high green emission was obtained at $2 \mathrm{~mol} \%$ as shown in Fig. S5 (see ESI†). Thus, the optimum concentrations of dopant and co-dopant were obtained: $\mathrm{Yb}^{3+} \sim 2 \mathrm{~mol} \%, \mathrm{Er}^{3+} \sim 1$ mol\% for $\mathrm{Y}_{2} \mathrm{O}_{3}: \mathrm{Yb}^{3+} / \mathrm{Er}^{3+}$ nanorods. The same optimum concentration of the sample also exhibits unique features in terms of downshift with a strong green emission at $562 \mathrm{~nm}$ upon excitation at $379 \mathrm{~nm}$. Furthermore, the energy level diagram of the optimum sample for the down-shift is shown in Fig. S6 (see ESI†). Furthermore, luminescent nanorods synthesized at the optimum concentration were fully characterized using several structural/microstructural and spectroscopic techniques.

The crystalline structure of $\mathrm{Y}_{2} \mathrm{O}_{3}: \mathrm{Yb}^{3+} / \mathrm{Er}^{3+}$ nanorods were investigated using $\mathrm{X}$-ray powder diffraction $\left(\mathrm{CuK \alpha}_{1}, \lambda=1.5406\right.$ $\AA)$. Fig. 1a shows the XRD pattern of $\mathrm{Y}_{2} \mathrm{O}_{3}: \mathrm{Yb}^{3+} / \mathrm{Er}^{3+}$ nanorods heat treated at $1000{ }^{\circ} \mathrm{C}$. The XRD results show that nanorods have a cubic phase with good crystallinity (JCPDS card no. 431036). The estimated lattice parameters for $\mathrm{Y}_{2} \mathrm{O}_{3}: \mathrm{Yb}^{3+} / \mathrm{Er}^{3+}$ nanorods were $a=b=c=(10.5869 \pm 0.0043) \AA$ which are comparable to standard lattice parameters $a=b=c=10.6040$ $\AA$. The inset of Fig. 1a shows the proposed crystal structure of $\mathrm{Y}_{2} \mathrm{O}_{3}$ where $\mathrm{Y}$ atoms are replaced by Er and $\mathrm{Yb}$ atoms. Fig. $1 \mathrm{~b}$ represents the Raman spectrum of luminescent nanorods. The Raman spectrum reveals that the host lattice has bands at 377 $\mathrm{cm}^{-1}, 428 \mathrm{~cm}^{-1}, 465 \mathrm{~cm}^{-1}, 511 \mathrm{~cm}^{-1}$ and $541 \mathrm{~cm}^{-1}$. The band present at $377 \mathrm{~cm}^{-1}$ represents the cubic phase of $\mathrm{Y}_{2} \mathrm{O}_{3}: \mathrm{Yb}^{3+}$ / $\mathrm{Er}^{3+}$ nanorods. The results obtained are in good agreement with the earlier reports for the Raman spectrum of the $\mathrm{Y}_{2} \mathrm{O}_{3}$ host. ${ }^{17}$ Fig. 1c demonstrates the Fourier-transform infrared (FT-IR) spectrum of nanorods sintered at $1000{ }^{\circ} \mathrm{C}$ as well as the assynthesized nanorods at $185^{\circ} \mathrm{C}$.

The FT-IR spectrum of the nanorods heated at $1000^{\circ} \mathrm{C}$ shows no peak around $3400 \mathrm{~cm}^{-1}$ because of the absence of the $\mathrm{OH}$
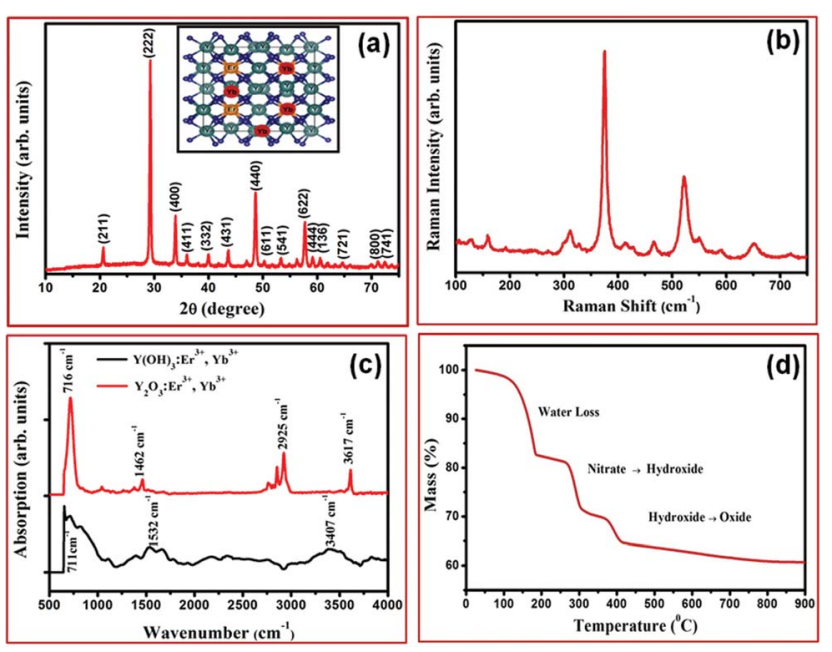

Fig. 1 (a) XRD pattern of $\mathrm{Y}_{2} \mathrm{O}_{3}: \mathrm{Yb}^{3+} / \mathrm{Er}^{3+}$ nanorods treated at $1000^{\circ} \mathrm{C}$. The right inset shows the proposed model of the cubic cell of the structure of $\mathrm{Y}_{2} \mathrm{O}_{3}: \mathrm{Yb}^{3+} / \mathrm{Er}^{3+}$ nanorods, (b) shows the Raman spectrum of $\mathrm{Y}_{2} \mathrm{O}_{3}: \mathrm{Yb}^{3+} / \mathrm{Er}^{3+}$ nanorods, (c) the $\mathrm{FT}$-IR spectrum of $\mathrm{Y}_{2} \mathrm{O}_{3}: \mathrm{Yb}^{3+} / \mathrm{Er}^{3+}$ nanorods and (d) results of the thermogravimetric analysis (TGA) of $\mathrm{Y}_{2} \mathrm{O}_{3}: \mathrm{Yb}^{3+} / \mathrm{Er}^{3+}$ nanorods. 
group, which suggests that the hydroxide formed $\left(\mathrm{Y}(\mathrm{OH})_{3}\right.$ :- $^{-}$ $\left.\mathrm{Yb}^{3+} / \mathrm{Er}^{3+}\right)$ at $185{ }^{\circ} \mathrm{C}$ during the hydrothermal process is completely converted into the oxide form $\left(\mathrm{Y}_{2} \mathrm{O}_{3}: \mathrm{Yb}^{3+} / \mathrm{Er}^{3+}\right)$ on sintering at $1000{ }^{\circ} \mathrm{C}$.

It is well known that the surface to volume ratio of the nanorod is larger than the spherical particle. The rare earth ions near the surface have large numbers of unsaturated bonds, which ultimately lead to surface defects and reaction with $\mathrm{OH}-$ groups. Consequently, the luminescence intensity of the assynthesized nanorods is lower than that of the heat treated nanorods. This indicates that the luminescence increases because of the absence of the $\mathrm{OH}$ group in the $\mathrm{Y}_{2} \mathrm{O}_{3}: \mathrm{Yb}^{3+} / \mathrm{Er}^{3+}$ nanorods. ${ }^{18}$ To understand the thermal decomposition of assynthesized $\mathrm{Y}_{2} \mathrm{O}_{3}: \mathrm{Yb}^{3+} / \mathrm{Er}^{3+}$ nanorods, the TGA investigation was carried out. Fig. 1d exhibits the TGA analysis of $\mathrm{Y}_{2} \mathrm{O}_{3}: \mathrm{Yb}^{3+} / \mathrm{Er}^{3+}$ nanorods, which demonstrates that the three temperature intervals corresponded to three substantial weight loss processes. The first interlude between $100-182{ }^{\circ} \mathrm{C}$ corresponds to water loss. The interlude between $270-310{ }^{\circ} \mathrm{C}$ is because of the decomposition of nitrates into hydroxides. The last interlude between $370{ }^{\circ} \mathrm{C}$ and $410{ }^{\circ} \mathrm{C}$ is because of the decomposition of hydroxide into oxide. The results obtained are consistent with results reported earlier. ${ }^{19}$

The surface morphology was examined by using SEM. Fig. 2a demonstrates the SEM image of nanorods. The image shows that the synthesized nanorods have a highly dense nanostructure with a uniform size distribution. The magnified version of the SEM image of the nanorods is shown in Fig. $2 b$ which clearly demonstrates the dimensions of the nanorods which are in the range of nanometers: 50-90 $\mathrm{nm}$ diameter and length $\sim 1-3 \mu \mathrm{m}$ at optimum growth conditions $\left(1000{ }^{\circ} \mathrm{C}\right.$ for 6 hours). The morphology of these luminescent materials originates as rod shapes because of the initial nucleation of the hexa-hydroxy phase formation during the intermediate reaction (after the hydrothermal process and before the final sintering at $1000{ }^{\circ} \mathrm{C}$ for oxide phase formation) where a nitrate solution of metals are converted into the hydroxy phase at $185^{\circ} \mathrm{C}$ as shown in Fig. S7 (see ESI $\dagger$ ). The surface morphologies of $\mathrm{Y}_{2} \mathrm{O}_{3}: \mathrm{Yb}^{3+} / \mathrm{Er}^{3+}$ nanorods at different time intervals during the growth at 1000 ${ }^{\circ} \mathrm{C}$ and statistical histogram of size distribution are shown in Fig. S8 and S9 (for more details, see the ESI $\dagger$ ). The different growth temperatures were employed to optimize the luminescence behaviour as well as the controlled morphologies. The EDAX analysis was performed for the element detection. Fig. 2c demonstrates the elemental analysis of nanorods, which confirmed the presence of $\mathrm{Er}, \mathrm{O}, \mathrm{Y}$ and $\mathrm{Yb}$ elements. The microstructural analysis of the synthesized nanorods was done using TEM. Fig. 2d shows the typical TEM micrograph of the nanorods. The TEM micrograph of an individual nanorod is shown in Fig. 2e. A typical HRTEM micrograph of a nanorod is shown in Fig. $2 \mathrm{f}$ and shows that the nanorods have well resolved lattice fringes without distortion which shows the good quality of the crystal. The estimated $d$-spacing of the nanorods is $\sim 0.302 \mathrm{~nm}$ which is comparable to the $0.306 \mathrm{~nm}$ corresponding to the (222) plane (JCPDS card no. 43-1036).

The PL and time-resolved spectroscopy were carried out to explore the spectroscopic features of the luminescent nanorods which legitimize its potential use for high-end security ink applications. The emission spectrum of $\mathrm{Y}_{2} \mathrm{O}_{3}: \mathrm{Yb}^{3+} / \mathrm{Er}^{3+}$ nanorods at $980 \mathrm{~nm}$ excitation is shown in Fig. 3a. The emission spectrum demonstrates that there are two emission bands: one band having a peak at $562 \mathrm{~nm}$ (green emission) and another at $660 \mathrm{~nm}$ (red emission). The green band corresponds to ${ }^{2} \mathrm{H}_{11 / 2}$, ${ }^{4} \mathrm{~S}_{3 / 2} \rightarrow{ }^{4} \mathrm{I}_{15 / 2}$ transition whereas the red emission band is a
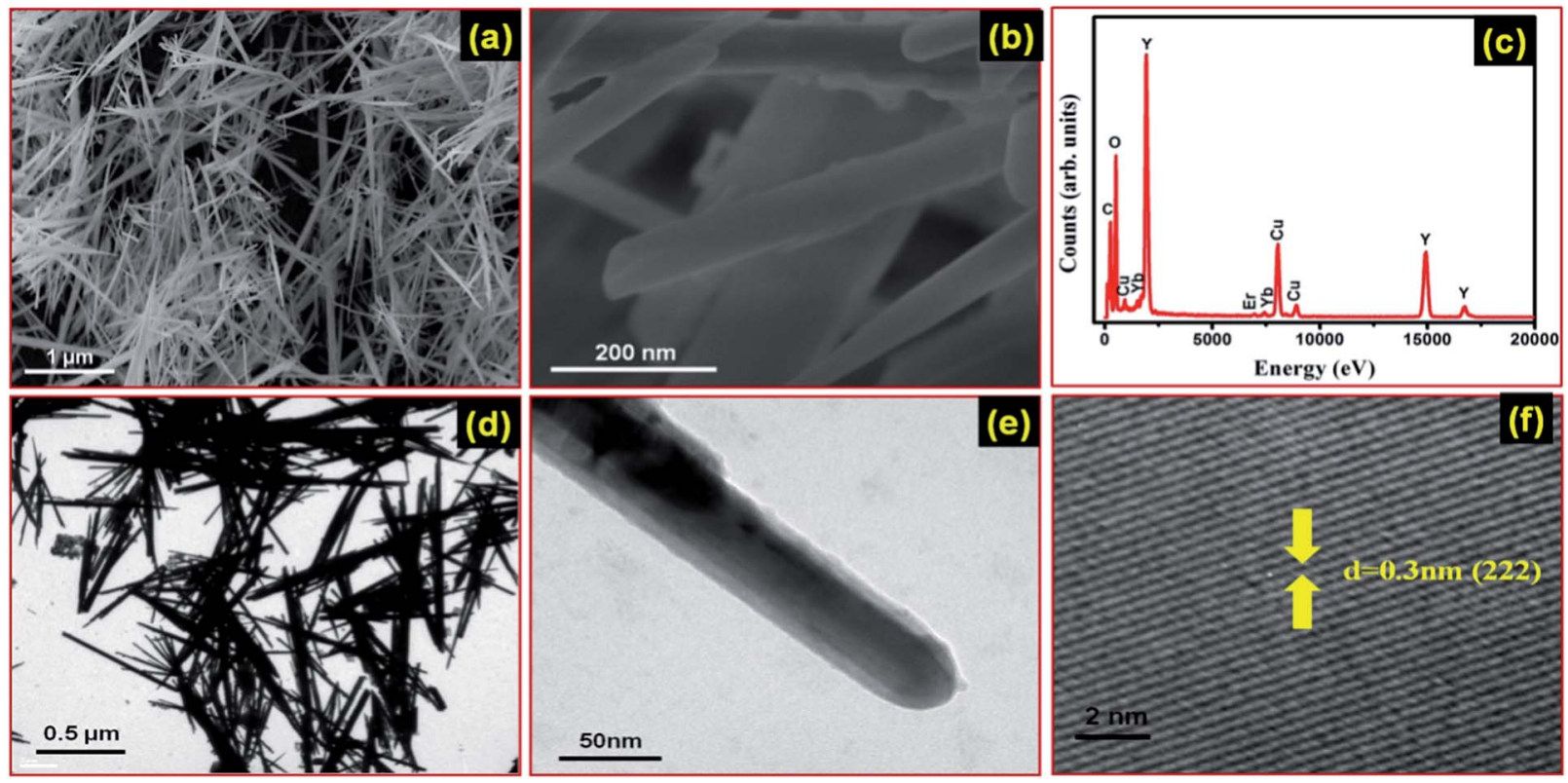

Fig. 2 (a) and (b) show the scanning electron microscopy (SEM) images of $\mathrm{Y}_{2} \mathrm{O}_{3}: \mathrm{Yb}^{3+} / \mathrm{Er}^{3+}$ nanorods and a magnified view of (a) respectively, (c) shows the results of the EDAX analysis of the $\mathrm{Y}_{2} \mathrm{O}_{3}: \mathrm{Yb}^{3+} / \mathrm{Er}^{3+}$ nanorods, (d) and (e) show the typical TEM image of nanorods and single nanorod, respectively, and (f) HRTEM results of $\mathrm{Y}_{2} \mathrm{O}_{3}: \mathrm{Yb}^{3+} / \mathrm{Er}^{3+}$ nanorod. 

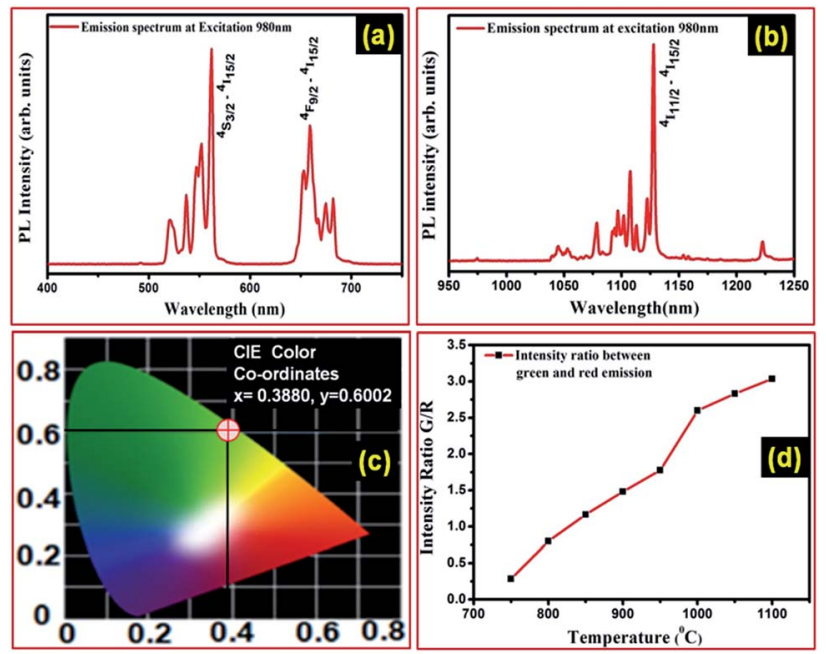

Fig. 3 (a) and (b) show the $\mathrm{PL}$ emission spectra of $\mathrm{Y}_{2} \mathrm{O}_{3}: \mathrm{Yb}^{3+} / \mathrm{Er}^{3+}$ nanorods at an excitation of $980 \mathrm{~nm}$, (c) demonstrates the CIE colour coordinates of green emission and (d) shows the ratio between green and red emission $\left(I_{G} / I_{R}\right)$ with temperature.

consequence of ${ }^{4} \mathrm{~F}_{9 / 2} \rightarrow{ }^{4} \mathrm{I}_{15 / 2}$ transition. The emission intensity of the green emission is greater than the red emission. The upconversion in $\mathrm{Y}_{2} \mathrm{O}_{3}: \mathrm{Yb}^{3+} / \mathrm{Er}^{3+}$ nanorods can be explained by two mechanisms, energy transfer and excited state absorption

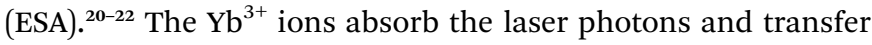
this energy to the $\mathrm{Er}^{3+}$ ion and because of this the population at ${ }^{4} \mathrm{I}_{11 / 2}$ increases. The populated ${ }^{4} \mathrm{I}_{11 / 2}$ level is further excited by three processes: ESA, energy transfer from the neighbouring $\mathrm{Er}^{3+}$ ion and energy transfer from the $\mathrm{Yb}^{3+}$ ion. The excited ${ }^{4} \mathrm{~F}_{7 / 2}$ level usually relaxes non-radiatively to two lower energy levels: ${ }^{2} \mathrm{H}_{11 / 2}$ and ${ }^{4} \mathrm{~S}_{3 / 2}$. The radiative emission from ${ }^{2} \mathrm{H}_{11 / 2}$ and ${ }^{4} \mathrm{~S}_{3 / 2}$ energy levels to the ground state ${ }^{4} \mathrm{I}_{15 / 2}$ produces the green emission. The populated ${ }^{4} \mathrm{~F}_{7 / 2}$ level also relaxes non-radiatively to ${ }^{4} \mathrm{I}_{13 / 2}$. The ${ }^{4} \mathrm{I}_{13 / 2}$ level is excited to the ${ }^{4} \mathrm{~F}_{9 / 2}$ level by the same mechanism as described previously. The ${ }^{4} \mathrm{~F}_{9 / 2}$ level relaxes radiatively which causes red emission. Fig. $3 \mathrm{~b}$ demonstrates the emission spectra at excitation at $980 \mathrm{~nm}$ in the NIR region. This result demonstrates that the $\mathrm{Y}_{2} \mathrm{O}_{3}: \mathrm{Yb}^{3+} / \mathrm{Er}^{3+}$ nanorods have a strong emission peak at $1127 \mathrm{~nm}$, corresponding to excitation at $980 \mathrm{~nm}$ which has not so far been reported in the literature. An additional, interesting feature of the $\mathrm{Y}_{2} \mathrm{O}_{3}: \mathrm{Yb}^{3+} / \mathrm{Er}^{3+}$ nanorods is shown in Fig. S10 (see ESI $\dagger$ ), where the emission spectrum exhibits the downshift characteristic of $\mathrm{Y}_{2} \mathrm{O}_{3}: \mathrm{Yb}^{3+} / \mathrm{Er}^{3+}$ nanorods in the NIR range of $1300-1700 \mathrm{~nm}$ at $980 \mathrm{~nm}$ excitation wavelength which has not so far been reported for this system. Fig. 3c shows the International Commission on Illumination (CIE) colour coordinates corresponding to emission at $980 \mathrm{~nm}$ with values of $x=0.3880$ and $y=0.6002$. The tuning of the emission wavelength from red to green is shown in Fig. $3 \mathrm{~d}$. Fig. 3d shows the intensity ratio of green to red emission $\left(I_{\mathrm{G}} / I_{\mathrm{R}}\right)$ at different temperatures. The green colour intensity is enhanced with increase in temperature leading to an increase in the intensity ratio.

Fig. 4a demonstrates the emission spectrum of nanorods having strong green emission at $564 \mathrm{~nm}$ which corresponds to
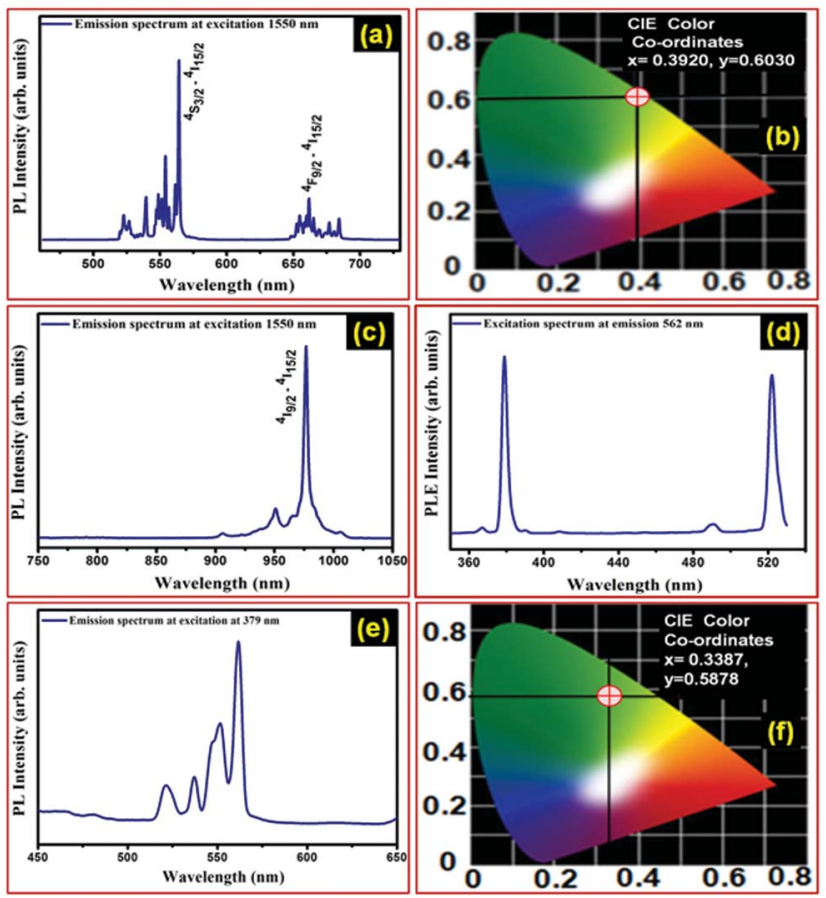

Fig. 4 (a) shows the $\mathrm{PL}$ emission spectrum $(460-730 \mathrm{~nm}$ visible region) of $\mathrm{Y}_{2} \mathrm{O}_{3}: \mathrm{Yb}^{3+} / \mathrm{Er}^{3+}$ nanorods at an excitation of $1550 \mathrm{~nm}$. (b) Represents the CIE colour coordinates of green emission of $\mathrm{Y}_{2} \mathrm{O}_{3}: \mathrm{Yb}^{3+} / \mathrm{Er}^{3+}$ nanorods at an excitation of $1550 \mathrm{~nm}$ in the visible region, (c) exhibits the $\mathrm{PL}$ emission spectrum of $\mathrm{Y}_{2} \mathrm{O}_{3}: \mathrm{Yb}^{3+} / \mathrm{Er}^{3+}$ nanorods at an excitation of $1550 \mathrm{~nm}$ in the NIR region (750-1050 $\mathrm{nm})$, (d) shows the excitation spectrum of $\mathrm{Y}_{2} \mathrm{O}_{3}: \mathrm{Yb}^{3+} / \mathrm{Er}^{3+}$ nanorods at an emission of $562 \mathrm{~nm}$ and (e) an emission spectrum of $\mathrm{Y}_{2} \mathrm{O}_{3}: \mathrm{Yb}^{3+} / \mathrm{Er}^{3+}$ nanorods at an excitation of $379 \mathrm{~nm}$ and (f) shows the $\mathrm{CIE}$ colour coordinates.

an excitation wavelength at $1550 \mathrm{~nm}$ which has not so far been reported in the literature. Fig. $4 \mathrm{~b}$ shows the corresponding CIE colour coordinates with values of $x=0.3920$ and $y=0.6030$. This emission provides additional high-end security in the visible range which has not been reported before for security ink applications. ${ }^{4,5}$ Fig. $4 \mathrm{c}$ exhibits the emission spectrum at excitation of $1550 \mathrm{~nm}$ in NIR region. The result demonstrates that the $\mathrm{Y}_{2} \mathrm{O}_{3}: \mathrm{Yb}^{3+} / \mathrm{Er}^{3+}$ nanorods have a strong emission peak at 976 $\mathrm{nm}$ which corresponds to excitation at $1550 \mathrm{~nm}$. The upconversion behavior of $\mathrm{Y}_{2} \mathrm{O}_{3}: \mathrm{Yb}^{3+} / \mathrm{Er}^{3+}$ nanorods at an excitation wavelength of $1550 \mathrm{~nm}$ is shown in Fig. S11 (see ESI†). Fig. 4d shows the excitation spectrum at an emission of $562 \mathrm{~nm}$. The emission of $\mathrm{Y}_{2} \mathrm{O}_{3}: \mathrm{Yb}^{3+} / \mathrm{Er}^{3+}$ nanorods at an excitation of $379 \mathrm{~nm}$ is shown in Fig. 4e, where a strong green emission can be easily seen which is required for the purposes of this security ink application. Fig. $4 \mathrm{f}$ demonstrates the CIE colour coordinates with values $x=0.3387$ and $y=0.5878$. Fig. S12 and S13 (see ESI $†$ ) show the emission spectra of $\mathrm{Y}_{2} \mathrm{O}_{3}: \mathrm{Yb}^{3+} / \mathrm{Er}^{3+}$ nanorods at excitation wavelengths of 488 and $522 \mathrm{~nm}$ which could also be utilized for the proposed application.

Furthermore, uniform emission from the $\mathrm{Y}_{2} \mathrm{O}_{3}: \mathrm{Yb}^{3+} / \mathrm{Er}^{3+}$ luminescent nanorod was examined by a fluorescent microscopic technique (Nikon ECLIPSE 80i microscope). Fig. $5 \mathrm{a}$ and b clearly show a high contrast, hypersensitive green and red 

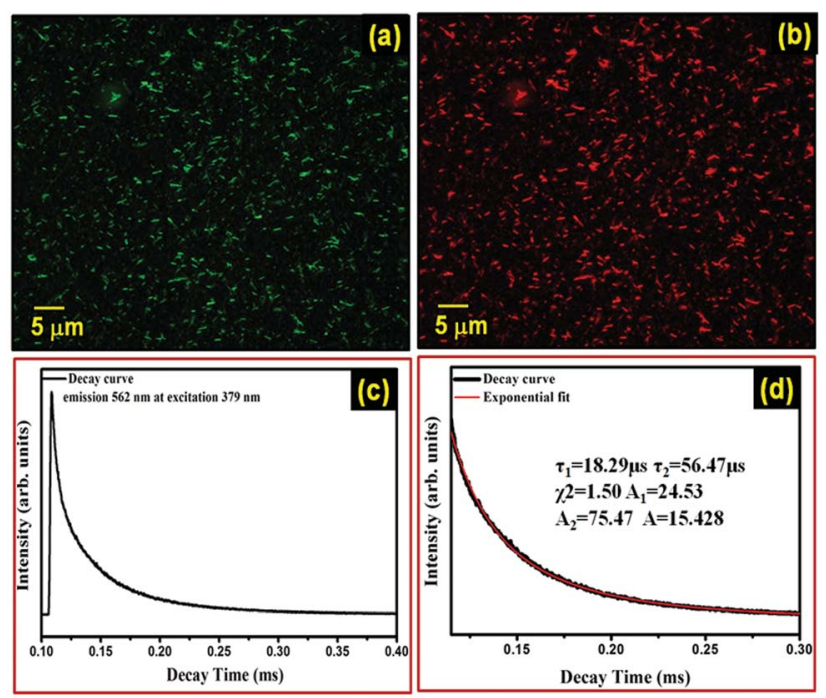

Fig. 5 (a) and (b) show the fluorescent images of $\mathrm{Y}_{2} \mathrm{O}_{3}: \mathrm{Yb}^{3+} / \mathrm{Er}^{3+}$ nanorods corresponding to the excitation wavelength of $375 \mathrm{~nm}$ and $980 \mathrm{~nm}$, respectively, (c) TRPL decay profile of $\mathrm{Y}_{2} \mathrm{O}_{3}: \mathrm{Yb}^{3+} / \mathrm{Er}^{3+}$ nanorods recorded at room temperature while monitoring the emission at $562 \mathrm{~nm}$, at an excitation wavelength of $379 \mathrm{~nm}$ and (d) exponential fitting of the decay profile which gives life time data and the parameters generated by the exponential fitting.

fluorescent image of $\mathrm{Y}_{2} \mathrm{O}_{3}: \mathrm{Yb}^{3+} / \mathrm{Er}^{3+}$ nanorods at excitation wavelengths of $375 \mathrm{~nm}$ and $980 \mathrm{~nm}$ (externally equipped diode lasers compatible with the Nikon ECLIPSE 80i microscope) without auto-fluorescence from the background. The fluorescent images obtained reveal that these nanorods exhibit the uniform spatial distribution of red and green colours from different points of the nanorods.

The time-resolved photoluminescence (TRPL) was recorded using a single photon counting technique with a microsecond xenon flash lamp as the source of the excitation. The decay life time is an important parameter for determining the performance of a luminescent material and its suitable potential applications. ${ }^{24,25}$ The efficiency of the radiative recombination depends on the decay time of the particular transition of the activator. ${ }^{25}$ The luminescence decay profile as well as the exponential fitting of the results obtained are shown in Fig. $5 \mathrm{c}$ and d. Fig. $5 \mathrm{c}$ exhibits the decay profile of $\mathrm{Y}_{2} \mathrm{O}_{3}: \mathrm{Yb}^{3+} / \mathrm{Er}^{3+}$ nanorods corresponding to excitation at $379 \mathrm{~nm}$ with emission of $562 \mathrm{~nm}$. The life time data obtained from the luminescent nanorods was best fitted to a double-exponential function as,

$$
I(t)=A_{1} \exp \left(-t / \tau_{1}\right)+A_{2} \exp \left(-t / \tau_{2}\right)
$$

where $\tau_{1}$ and $\tau_{2}$ are the decay life times of the luminescence, and $A_{1}$ and $A_{2}$ are the weighting parameters. Fig. 5d demonstrates the exponential fitting of decay profile as described in eqn (1). The parameters generated from fitting are listed in the inset in Fig. 5 d. The observed life times are $\tau_{1} \sim 18.29 \mu$ s and $\tau_{2} \sim 56.47$ $\mu \mathrm{s}$. For double exponential decay, the average life time, $\tau_{\mathrm{av}}$ is usually tailored to substitute the various components of the luminescence life time, which is determined by the following equation: $:^{23,24}$

$$
\tau_{\mathrm{av}}=\left(A_{1} \tau_{1}^{2}+A_{2} \tau_{2}^{2}\right) /\left(A_{1} \tau_{1}+A_{2} \tau_{2}\right)
$$

The average life time of $\mathrm{Y}_{2} \mathrm{O}_{3}: \mathrm{Yb}^{3+} / \mathrm{Er}^{3+}$ nanorods is calculated as $\tau_{\mathrm{av}} \sim 52.83 \mu \mathrm{s}$. The result obtained is highly suitable for the proposed security ink applications as well as many other optical display applications.

Finally, the transparent multi-stage excitable security inks application of $\mathrm{Y}_{2} \mathrm{O}_{3}: \mathrm{Yb}^{3+} / \mathrm{Er}^{3+}$ nanorods for anti-counterfeiting applications, which was not reported earlier, was demonstrated. ${ }^{25-27}$ The main advantages of using standard gold PVC medium for the dispersion of luminescent nanorods are its easy availability in the market at a low cost, convenience of printing, highly stability and the sticky nature of medium (viscosity $\sim 3000 \mu \mathrm{P}$ ) with paper which is highly desirable for such applications. For the successful demonstration of anti-counterfeiting applications, first the luminescent security ink was synthesized. For this purpose, specific amount of luminescent nanorods was incorporated in PVC gold medium. The ratio of luminescent nanorod powder and PVC gold medium has been described in detail in the experimental section. In order to examine the dispersion of $\mathrm{Y}_{2} \mathrm{O}_{3}: \mathrm{Yb}^{3+} / \mathrm{Er}^{3+}$ nanorods in PVC gold medium, the transparent security ink solution was excited by a $980 \mathrm{~nm}$ wavelength diode laser which can be tuned to a maximum of $0-$ $6 \mathrm{~W}$, as shown in Fig. S14 (see ESI†). An SEM image of the sample upon its dispersion into PVC gold medium was also taken as shown in Fig. S15 (see ESI†). The synthesized ink is highly stable for several hours and also maintains its optical transparency with higher luminescence. Furthermore, for the printing of security codes, a standard screen printing technique was used. The detailed systematic process of screen printing on black paper in the present investigations is shown in Fig. 6a. The image of Mahatma Gandhi, alphabets and circular spots were printed on black paper to demonstrate the anti-counterfeiting applications. Fig. $6 \mathrm{~b}$ demonstrates the printed pattern under different excitation wavelengths (379 and $980 \mathrm{~nm}$ ). The pattern is printed on black paper using transparent security ink synthesized in the present investigation which can be easily seen in Fig. 6b. The visibility of the printed item is almost negligible in normal light. When the printed patterns (circular spots) were excited under a $980 \mathrm{~nm}$ laser light, they showed red and green coloured emissions. Furthermore, when other printed patterns (a sketched image of Mahatma Gandhi and alphabets) were exposed to an excitation wavelength of $379 \mathrm{~nm}$, a green coloured emission was exhibited which can be easily seen at the right-hand side of Fig. 6b. The fluorescent mapping of the selected area of the printed luminescent alphabets was further examined to validate the distribution of the $\mathrm{Y}_{2} \mathrm{O}_{3}: \mathrm{Yb}^{3+}$ / $\mathrm{Er}^{3+}$ nanorods in PVC gold medium. Fig. 6c shows the PL mapping of that particular area upon excitation by wavelengths of 375, 980 and $1550 \mathrm{~nm}$ as shown in Fig. 6c (i), (ii) \& (iii), respectively, together with their corresponding PL spectra. The clear evidence of PL and PL mapping at various wavelengths prove its potential use for multi-stage security ink applications to prevent counterfeiting. Finally, a realistic scheme was 

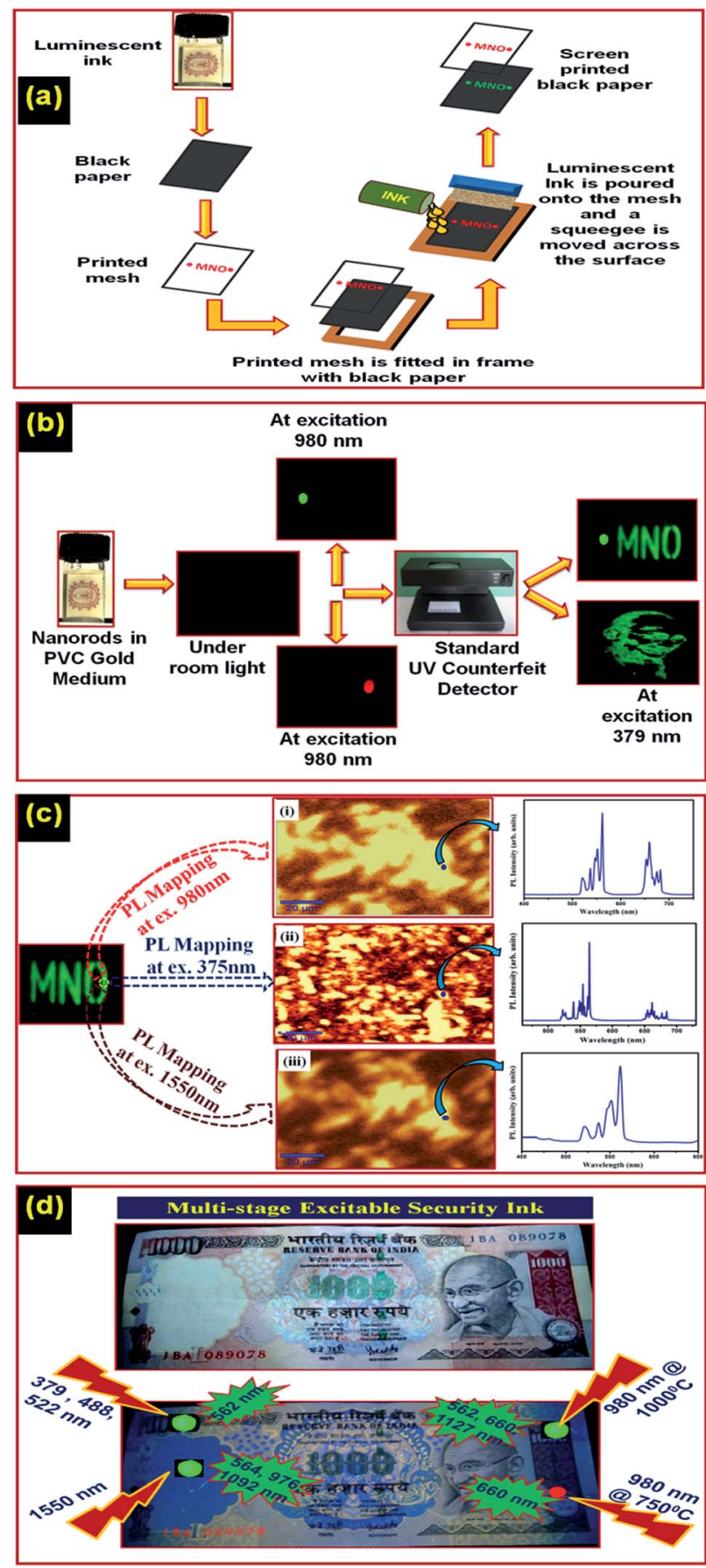

Fig. 6 (a) Demonstrates various steps involved in the systematic process for screen printing on black paper, (b) printed pattern images of Mahatma Gandhi and alphabets exposed to a $379 \mathrm{~nm}$ excitation wavelength, and it can be easily seen that these images clearly show the strong green colour emission and the images are clearly visible, (c) the PL mapping image of the selected region of printed alphabets excited with multi-stage excitation wavelengths at 375, 980 and 1550 $\mathrm{nm}$ as shown in (i), (ii) and (iii) and their PL spectra, respectively and (d) the proposed scheme for high-end protection of Indian currencies to protect counterfeiting.

demonstrated on the basis of the obtained spectroscopic results which is a legitimate proof of its application for high-end protection of Indian currency as shown in Fig. 6d. Concisely, the results obtained reveal that $\mathrm{Y}_{2} \mathrm{O}_{3}: \mathrm{Yb}^{3+} / \mathrm{Er}^{3+}$ nanorods have good structural properties, optical properties, excellent solubility in PVC gold medium and can be easily produced at a large scale for commercial printing applications. The results achieved reveal that $\mathrm{Y}_{2} \mathrm{O}_{3}: \mathrm{Yb}^{3+} / \mathrm{Er}^{3+}$ nanorods can be the ultimate choice for a multi-stage security ink application for anti-counterfeiting. Therefore, we can expect $\mathrm{Y}_{2} \mathrm{O}_{3}: \mathrm{Yb}^{3+} / \mathrm{Er}^{3+}$ nanorods to offer a new perspective for the fabrication of low-cost multi-stage security ink for advanced anti-counterfeiting applications.

\section{Conclusions}

In summary, the development of a novel, multi-stage excitable $(379 \mathrm{~nm}, 980 \mathrm{~nm}$ and $1550 \mathrm{~nm}$ ) and highly-luminescent $\mathrm{Y}_{2} \mathrm{O}_{3}: \mathrm{Yb}^{3+} / \mathrm{Er}^{3+}$ nanorod based transparent $(\sim 81 \%)$ security ink has been successfully demonstrated. These luminescent nanorods were synthesized using a hydrothermal method which can be scaled up in large quantity and it could emit both hypersensitive green and strong red in a single host lattice at different excitation wavelengths. Furthermore, these nanorods have exhibited both down-shift as well as up-conversion features at a single excitation NIR wavelength of $980 \mathrm{~nm}$. Furthermore, these luminescent nanorods have exhibited the striking features related to the tuning of emission colours from red to green by controlling the sintering temperature. Thus, the integration of luminescent nanorods with a suitable PVC gold medium offers new opportunities for high-end multi-stage excitable transparent security ink which is highly useful for protection against counterfeiting of important documents as well as for currency.

\section{Acknowledgements}

The authors wish to thank Prof. R. C. Budhani, Director, NPL, New Delhi, for his keen interest in the work. The authors are thankful to Prof. O. N. Srivastava (Banaras Hindu University, Varanasi) for his encouragement. Mr Pawan Kumar gratefully acknowledges the University Grant Commission (UGC), Government of India, for financial assistance under the RGNF Research Fellowship, Award no. F1-17.1/2011-12/R GNF-SCPUN-12604/(SA-III/Website).

\section{Notes and references}

1 B. L. Volodin, B. Kippelen, K. Meerholz, B. Javidi and N. Peyghambarian, Nature, 1996, 83, 58-60.

2 B. K. Gupta, D. Haranath, S. Saini, V. N. Singh and V. Shanker, Nanotechnology, 2010, 21, 055607.

3 S. Armstrong, O. Graydon, D. Pile and R. Won, Nat. Photonics, 2012, 6, 801.

4 W. J. Kim, M. Nyk and P. N. Prasad, Nanotechnology, 2009, 20, 185301.

5 J. M. Meruga, W. M. Cross, P. S. May, Q. Luu, G. A. Crawford and J. J. Kellar, Nanotechnology, 2012, 23, 395201.

6 C. E. Chesak, Opt. Commun., 1995, 115, 429-436.

7 J. Janucki and J. Owsik, Opt. Commun., 2003, 228, 63-69.

8 A. K. Aggarwal, S. K. Kaura, D. P. Chhachhia and A. K. Sharma, Opt. Laser Technol., 2006, 38, 117-121. 
9 B. Yoon, J. Lee, I. S. Park, S. Jeon, J. Lee and J. M. Kim, J. Mater. Chem. C, 2013, 1, 2388-2403.

10 Y. Cui, R. S. Hegde, I. Y. Phang, H. K. Lee and X. Y. Ling, Nanoscale, 2014, 6, 282-288.

11 K. Huang, J. M. Carulli and Y. Makris, International Conference IEEE, 2013.

12 A. K. Deisingh, Analyst, 2005, 130, 271-279.

13 A. Mills, Chem. Soc. Rev., 2005, 34, 1003-1011.

14 R. Abargues, P. J. Rodriguez-Canto, S. Albert, I. Suarezb and P. Martínez-Pastor, J. Mater. Chem. C, 2014, 2, 908-915.

15 V. K. Rai, A. Pandey and R. Dey, J. Appl. Phys., 2013, 113, 083104.

16 A. P. Singh, B. K. Gupta, M. Mishra, Govind, A. Chandra, R. B. Mathur and S. K. Dhawan, Carbon, 2013, 56, 86-96.

17 A. Ubaldini and M. M. Carnasciali, J. Alloys Compd., 2008, 454, 374-378.

18 H. Guo and Y. M. Qiao, Opt. Mater., 2009, 31, 583-589.

19 B. Tang, J. Ge, C. Wu, L. Zhuo, J. Niu, Z. Chen, Z. Shi and Y. Dong, Nanotechnology, 2004, 15, 1273-1276.
20 X. Li, Q. Li, J. Wang and J. Li, J. Lumin., 2007, 124, 351-356. 21 L. Yanhoung, Z. Yongming, H. Guangyan and Y. Yongning, J. Rare Earths, 2008, 26, 450-454.

22 A. Martíneza, J. Moralesa, P. Salasb, C. Angeles-Chávezb, L. A. Díaz-Torresc and E. De la Rosa, Microelectron. J., 2008, 39, 551-555.

23 B. K. Gupta, N. N. Tharangattu, S. A. Vithayathil, Y. Lee, S. Koshy, A. L. M. Reddy, A. Saha, V. Shanker, V. N. Singh, B. A. Kaipparettu, A. A. Martí and P. M. Ajayan, Small, 2012, 8, 3028-3034.

24 B. K. Gupta, T. Palanisamy, N. N. Tharangattu, L. Song, G. Wei, H. Takuya, A. L. M. Reddy, A. Saha, V. Shanker, M. Endo, A. A. Martí and P. M. Ajayan, Nano Lett., 2011, 11, 5227-5533.

25 Y. Liu, K. Ai and L. Lu, Nanoscale, 2011, 3, 4804-4810.

26 J. Wang, T. Wei, X. Li, B. Zhang, J. Wang, C. Huang and Q. Yuan, Angew. Chem., Int. Ed., 2014, 53, 1616-1620.

27 Y. Zhang, L. Zhang, R. Deng, J. Tian, Y. Zong, D. Jin and X. Liu, J. Am. Chem. Soc., 2014, 136, 4893-4896. 